\title{
Association Between Metformin and Sulfonylurea Monotherapies and Cancer Incidence: A Real-World Cohort Study in Shanghai, China
}

\author{
Jing-Hong Ye $\cdot$ Meng-Hua Qian · Li-Zheng Shi $\cdot$ Lu Ye
}

Received: October 21, 2018 / Published online: January 8, 2019

(C) The Author(s) 2019

\begin{abstract}
Introduction: Previous studies have shown that patients with type 2 diabetes mellitus have an increased risk of cancer. The use of antidiabetic medication (ADM) may play an important role in the cancer development. The relationship between oral ADM and cancer incidence has not been investigated in type 2 diabetes mellitus patients in mainland China yet.

Methods: A community-based diabetes cohort was extracted from the Shanghai Community Diabetes Management System database, which is a patient registry from general practices. The cohort included 2353 newly onset type 2 diabetes mellitus patients from 2006 to 2010 aged 35 years or more. Patients were grouped into
\end{abstract}

Enhanced Digital Features To view enhanced digital features for this article go to https://doi.org/10.6084/ m9.figshare.7466744.

J.-H. Ye · L. Ye $(\bowtie)$

Department of Health Economics, School of Public Health, Key Lab of Health Technology Assessment, National Health Commission, Fudan University,

Shanghai, China

e-mail: lye@fudan.edu.cn

J.-H. Ye · M.-H. Qian

Shanghai Hongkou Center for Disease Control and Prevention, Shanghai, China

\section{L.-Z. Shi $(\bowtie)$}

Tulane University of School and Public Health and Tropical Medicine, New Orleans, LA, USA

e-mail: lshi1@tulane.edu nonusers of antidiabetic medication $(n=722)$, metformin monotherapy $(n=374)$, sulfonylurea monotherapy $(n=653)$, metformin and sulfonylurea combination therapy $(n=302)$, and other medication therapies $(n=302)$ on the basis of initial treatment type at registry entry. Cancer incidence was identified from the Shanghai Cancer Registry Organization. Comparisons between monotherapy and nonuser of medication were conducted using Cox proportional hazards models.

Results: A total of 94 cancer cases were identified during 5 years median follow-up. Compared with nonusers, sulfonylurea monotherapy was associated with significantly lower risk of cancer [adjusted HR $=0.50$ (95\% CI 0.29-0.85)] whereas risk was $49 \%$ lower with metformin monotherapy [adjusted $\mathrm{HR}=0.51$ (95\% CI 0.27-0.99)].

Conclusion: The real-world evidence suggested that the use of metformin or sulfonylurea was associated with lower risk of cancer incidence in a cohort of newly onset type 2 diabetes mellitus patients.

Keywords: Cancer incidence; Chinese; Diabetes; Metformin; Sulfonylurea

\section{INTRODUCTION}

Type 2 diabetes mellitus (T2DM) is a global epidemic with more than 415 million patients 
now and 642 million patients predicted by 2040 [1]. Cancer is a leading cause of death globally, with an expected $70 \%$ increase in new cases over next two decades [2]. Since strong evidence from several epidemiological studies proved that T2DM is a risk factor for cancer [3] and increases cancer mortality [4]. Among patients with diabetes, hyperinsulinemia and hyperglycemia which may be mediated through metabolic inflammation may play important roles in the development of cancer $[5,5]$.

The relationship between antidiabetic medication (ADM) and cancer was also explored. As the first-line ADM, metformin's effect on relieving insulin resistance and inflammation may lead to potential antineoplastic action, modifying the insulin-like growth factor (IGF) signaling system and adenosine monophosphate activated protein kinase (AMPK) pathways [7, 8]. Meta-analyses of population observational studies indicated that metformin use is associated with reduced risk of cancer development compared with other ADMs [9]. The findings were consistent with randomized controlled trials (RCTs) [10, 11]. Other metaanalyses attributed the effect of metformin on cancer to biases and confounders [12].

Sulfonylureas are the most widely used oral ADM in China [13]. Meta-analyses showed that sulfonylurea use is associated with an increase in all-cancer risk [9]; however, the relationship failed to be confirmed by RCTs [10]. Moreover, some studies found that the risk of cancer may not be equivalent with all generations of sulfonylureas [14]. Furthermore, evidence from biological studies showed that glipizide may tend to suppress tumor development by inhibiting angiogenesis and embryonic vasculogenesis $[15,16]$.

Studies of the association between oral ADMs and cancer incidence in T2DM populations in mainland China are still lacking, despite the huge T2DM population and the widespread use of oral ADMs in China. The objective of this study was to examine the association between monotherapy of metformin or sulfonylurea and cancer incidence using a community population-based retrospective cohort in newly onset T2DM validated from the Shanghai Community Diabetes Management System of China.

\section{METHODS}

\section{Data Source}

The Shanghai Community Diabetes Management System in Hongkou District was established in 2006 with more than 25,000 diabetes patients from all eight community health service centers of Hongkou District as of 2015. The management system provided free health services including lifestyle intervention, health education, medication management, and monitoring of diabetes and its complications by general physicians (GP) though both outpatient clinics and visits regularly. It collected information on medication of T2DM, diet and physical activity, anthropometric measurements, and behavior monitoring.

\section{Study Population}

We extracted 2545 patients with newly onset T2DM who were enrolled in the system from 1 January 2006 and 31 December 2010 (Fig. 1). The study cohort was 2353 patients aged 35 years or more without cancer history, ADM history, and history of microvascular and macrovascular complications. To avoid the protopathic-type bias, high-quality registry data from the Shanghai Cancer Registry Organization allowed the effective exclusion of patients with a cancer history at baseline, developing cancer at the first follow-up year, diagnoses of metastatic cancer and secondary cancer, as well as using insulin as first prescription.

All procedures performed in studies involving human patients were in accordance with the ethical standards of the institutional and/or national research committee and with the 1964 Helsinki declaration and its later amendments or comparable ethical standards. Informed consent was obtained from all individual patients included in the study. Ethical approval was given by the medical ethics committee of Fudan University, School of Public Health, with the following reference numbers: IRB00002408 and FWA00002399. 


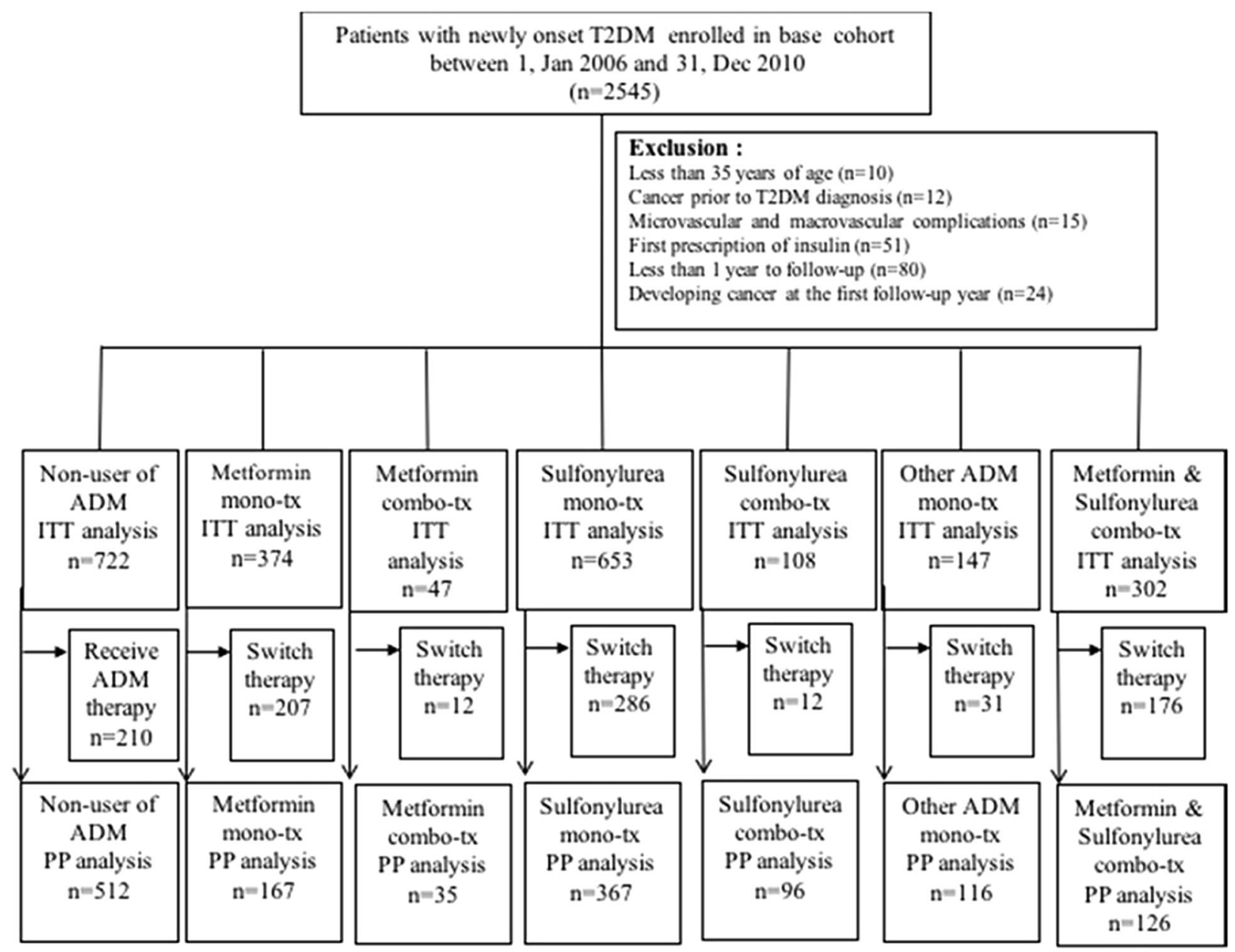

Fig. 1 Following the selection criteria for the study cohort, 2353 newly onset type 2 diabetes mellitus patients were extracted and categorized into seven subgroups by the initial therapy (ITT analysis). Patients who retained initial

\section{Exposure Definition}

Exposure to ADM was defined on the basis of the records of the Shanghai Community Diabetes Management System in Hongkou District. In China, both metformin and sulfonylurea are first-line treatments for T2DM patients [17]. We defined the first prescription date as the index date, which should be less than 6 months from diagnosis date. The study cohort was categorized into the following seven mutually exclusive groups according to the initial therapy which lasted for more than a 1-year treatment period: therapy during the follow-up were allocated to the perprotocol (PP) cohort. Mono-tx, monotherapy; Combo-tx, combination therapy

1. Nonuser of ADM, i.e., patients who did not receive any $\mathrm{ADM}$ prescriptions.

2. Metformin monotherapy.

3. Sulfonylurea monotherapy, including the second generation (glibenclamide, glibornuride, gliclazide, glipizide, gliquidone) and the third generation (glimepiride).

4. Monotherapy of other oral ADMs, including $\alpha$-glucosidase inhibitors (acarbose), meglitinide analogues (nateglinide, repaglinide), thiazolidinediones (rosiglitazone, pioglitazone), glucagon-like peptide-1 (GLP-1) receptor antagonists (exenatide), dipeptidyl peptidase-4 (DPP4) inhibitors (sitagliptin, vildagliptin). 
5. Combination therapy of sulfonylurea and metformin if patients were prescribed the combination therapy as initial treatment.

6. Combination therapy of sulfonylurea and other oral ADMs if patients were prescribed sulfonylurea and other oral ADMs together as initial treatment and no exposure to metformin is required.

7. Combination therapy of metformin and other oral ADMs if patients were prescribed metformin and other oral ADMs together as initial treatment and no exposure to sulfonylurea was required.

\section{Outcome Assessment}

The outcome of study was the first diagnosis of primary cancer which was identified from the Shanghai Cancer Registry Organization using International Classification of Diseases, 10th revision (ICD-10) code (C00-C97, D00-D09), including the following data: hospital of diagnosis, date of diagnosis, diagnostic methods, and tumor lymph node metastasis (TNM) classification of cancer. Mortality cases were identified from the Shanghai Death Record System to confirm the causes of death.

\section{Covariates}

Socioeconomic-demographic characteristics, lifestyle, ADM, anthropometric measurements, and incidence related to T2DM were collected from patient registry via the GP's visit every 3 months. Physical activity was converted to metabolic equivalent of task (MET) hours per day $($ MET $\times$ h/day) [18]. Smoking status was categorized into never use and ever use. Excessive alcohol consumption was converted to pure alcohol, defined as more than $168 \mathrm{~g}$ per week for men and more than $84 \mathrm{~g}$ per week for women in the last year. The difference between the baseline fasting plasma glucose (FPG) and the average FPG in the first following year (i.e., DFPG = baseline FPG minus mean FPG in the first following year) was used to represent the primary effect of different ADM strategies. Cox proportional hazard was analyzed with the stratification of common risk factors for cancer, including age $(\geq 65,<65)$, body mass index (BMI) $\left(\geq 24,<24 \mathrm{~kg} / \mathrm{m}^{2}\right)$, waist circumstance (male $\geq 90 \mathrm{~cm}$ or female $\geq 80 \mathrm{~cm}$; male $<90$ $\mathrm{cm}$ or female $<80 \mathrm{~cm})$, FPG $(\geq 7.2,<7.2$ $\mathrm{mmol} / \mathrm{L}$ ) [19], adherence (non-switching or switching of therapy during follow-up), smoking status (never, ever), and excessive alcohol assumption (never, ever).

\section{Statistical Analysis}

Considering the time-related bias in observational studies, intention-to-treat (ITT) could be a suitable approach to realize "observational studies analyzed like randomized experiments" [20]. In the ITT group, patients with monotherapy of sulfonylurea, metformin, and other ADMs or with combination therapy were compared with patients without any ADM therapy respectively. Patients were allocated to the group as the initial therapy irrespective of subsequent change in therapy.

In addition, the per-protocol (PP) analysis was conducted to examine the results. Throughout the follow-up duration, patients who withdrew the allocation of initial therapy were excluded from analysis (see Fig. 1).

Descriptive analyses were conducted for the whole cohort and by ADM groups. The Kaplan-Meier method was used to estimate the cumulative incidence of cancer in each group, and Cox proportional hazard models were conducted to assess the differences between groups. Crude hazard ratio (HR) and 95\% CI of the effectiveness of ADM groups were compared with those of non-ADM users. Furthermore, multivariable Cox proportional hazard regressions were used adjusting for age, gender, baseline BMI, FPG (pre-prandial capillary plasma glucose), hypertension, smoking status, and excessive alcohol consumption. A sensitivity analysis was performed among monotherapy medication groups, i.e., nonuser of ADM, monotherapy of metformin, and monotherapy of sulfonylurea. The statistical analyses were performed using SPSS version 16.0. Two-sided test of $P<0.05$ was considered a significant difference. 


\section{RESULTS}

\section{Baseline Characteristics}

A total of 2353 newly onset cases of T2DM were identified and categorized according to the initial therapy of the first year: no use of medication $\quad(n=722, \quad 30.68 \%), \quad$ sulfonylurea monotherapy $(n=653,27.75 \%)$, metformin monotherapy ( $n=374,15.89 \%)$, sulfonylurea and metformin combination therapy $(n=302$, $12.83 \%)$, other ADMs $(n=147,6.25 \%)$, sulfonylurea combined other ADMs $(n=108$, $4.59 \%)$, and metformin combined other ADMs $(n=47,2.00 \%)$. Patients with insulin as initial therapy were excluded because they likely had long-standing T2DM. As of the end of followup, 1419 patients retained the location of initial therapy as the (PP cohort, 167 patients retained metformin monotherapy, 367 patients retained sulfonylurea monotherapy, 126 patients retained metformin and sulfonylurea combination therapy, and 512 patients retained nonuser of ADM till the end of follow-up. Baseline and exposure characteristics are presented among nonusers of ADM and ADM groups in ITT and PP cohorts, respectively. The mean age of ITT cohort at baseline was $67.3 \pm 11.0$ years, and $57.7 \%$ of patients were female. The mean BMI was $24.5 \pm 3.2 \mathrm{~kg} / \mathrm{m}^{2}$ and mean FPG was $7.4 \pm 1.6 \mathrm{mmol} / \mathrm{L}$. Compared with the nonuser of ADM group, patients with ADM were more likely to have higher BMI, FPG level, and unhealthy lifestyle (all $p<0.05$ ) (Table 1 ). In the PP cohort, compared to the nonuser of ADM group, the metformin and sulfonylurea combination group was more likely be younger and less healthy. Except for a higher level of FPG (all $p<0.05)$, other ADM groups had similar characteristics at baseline (Table 2).

After 12 months, the mean FPG of the ITT cohort was $6.8 \pm 1.1 \mathrm{mmol} / \mathrm{L}$ and the improvement (DFPG) compared with the baseline FPG was $0.5 \pm 1.7 \mathrm{mmol} / \mathrm{L}$ in the ITT cohort. Compared to the nonuser of ADM group, the sulfonylurea combination group and the metformin and sulfonylurea combination group were more likely to have a better control of FPG (all $p<0.05$ ) (Table1). In the PP cohort, compared to the nonuser of ADM group, the metformin monotherapy group was more likely to have a better control of FPG $(p<0.05)$ (Table 2).

\section{Association of Metformin and Sulfonylurea Therapy with Cancer Incidence}

During 5 years follow-up, a total of 94 new cancer cases were diagnosed. Cancer of the lung, colorectum, breast, prostate, and thyroid accounted for the majority of cases in both ITT and PP cohorts (Table 3).

In the ITT cohort, compared with the nonusers of ADM, the sulfonylurea monotherapy group was associated with a significant decreased risk of any cancer (11.53 vs. 6.27 per 1000 person-years, respectively, HR 0.55; 95\% CI 0.32-0.93). The cancer incident risk of other medication ITT group without metformin or sulfonylurea was similar to the nonuser of ADM group (11.48 vs. 11.53 per 1000 person-years, respectively, HR 1.00; 95\% CI 0.47-2.13). Considering risk factors and potential confounders of cancer incidence, the adjusted HRs were 0.50 (95\% CI 0.29-0.85), 0.51 (95\% CI 0.27-0.99), and 0.39 (95\% CI $0.18-0.84)$ for sulfonylurea monotherapy, metformin therapy, and metformin and sulfonylurea combination therapy, respectively (Table 4 ).

In the PP cohort, a total of 60 first incident cancers were retained on the original allocation of therapy. Compared with the nonuser of ADM group, the metformin monotherapy group was associated with a significant decreased risk of any cancer (12.27 vs. 3.73 per 1000 personyears, respectively, HR 0.31; 95\% CI 0.09-1.00); the sulfonylurea monotherapy group was associated with a borderline significantly lower cancer incident (12.27 vs. 6.15 per 1000 personyears, respectively, HR 0.51; 95\% CI 0.25-1.01). After adjustment for age, gender, BMI, FPG, DFPG, physical activity, smoking, and excessive alcohol assumption, the adjusted HRs were 0.29 (95\% CI 0.09-0.96) and 0.45 (95\% CI 0.22-0.91) for metformin and sulfonylurea monotherapy groups, respectively (Table 4). 


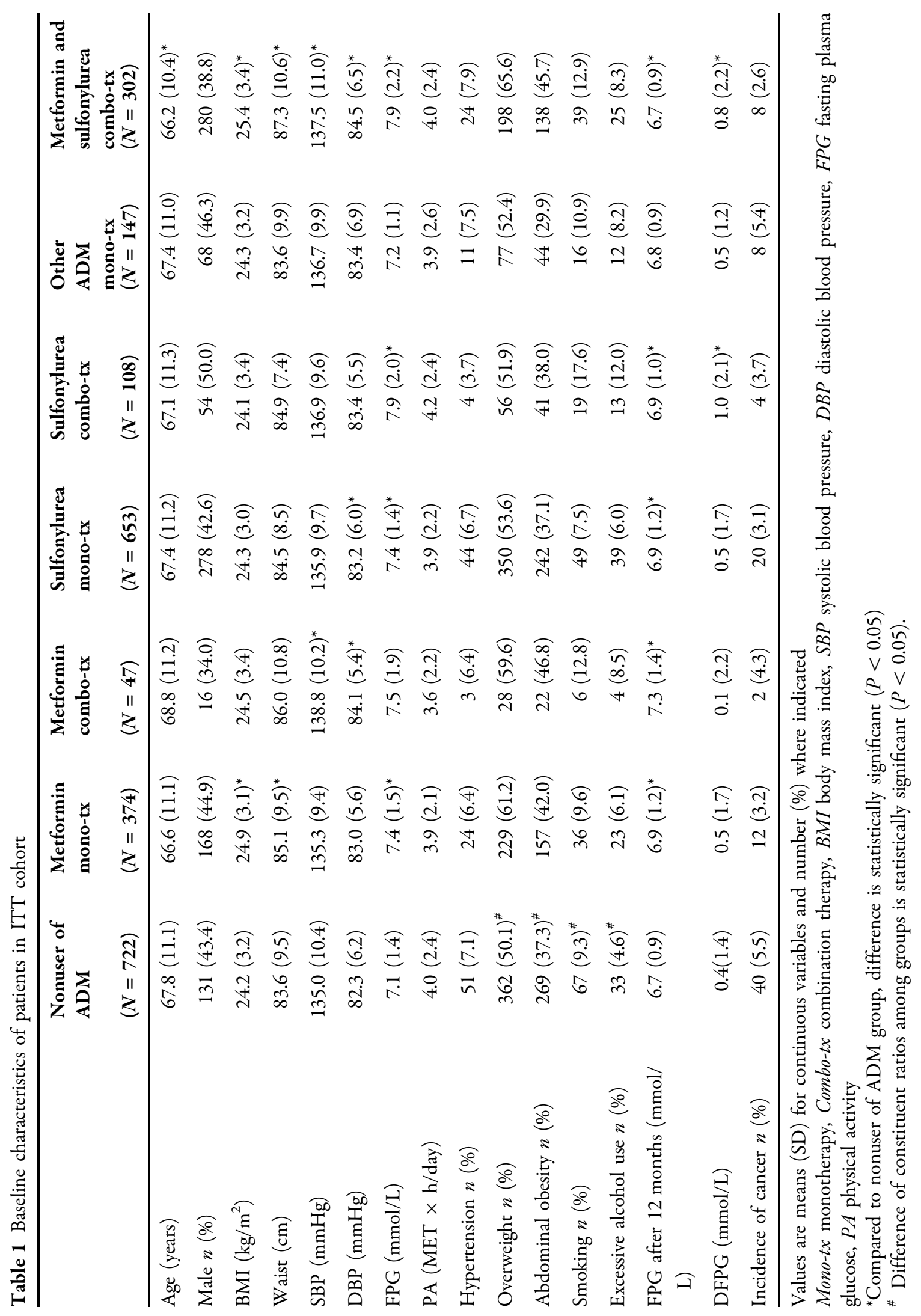




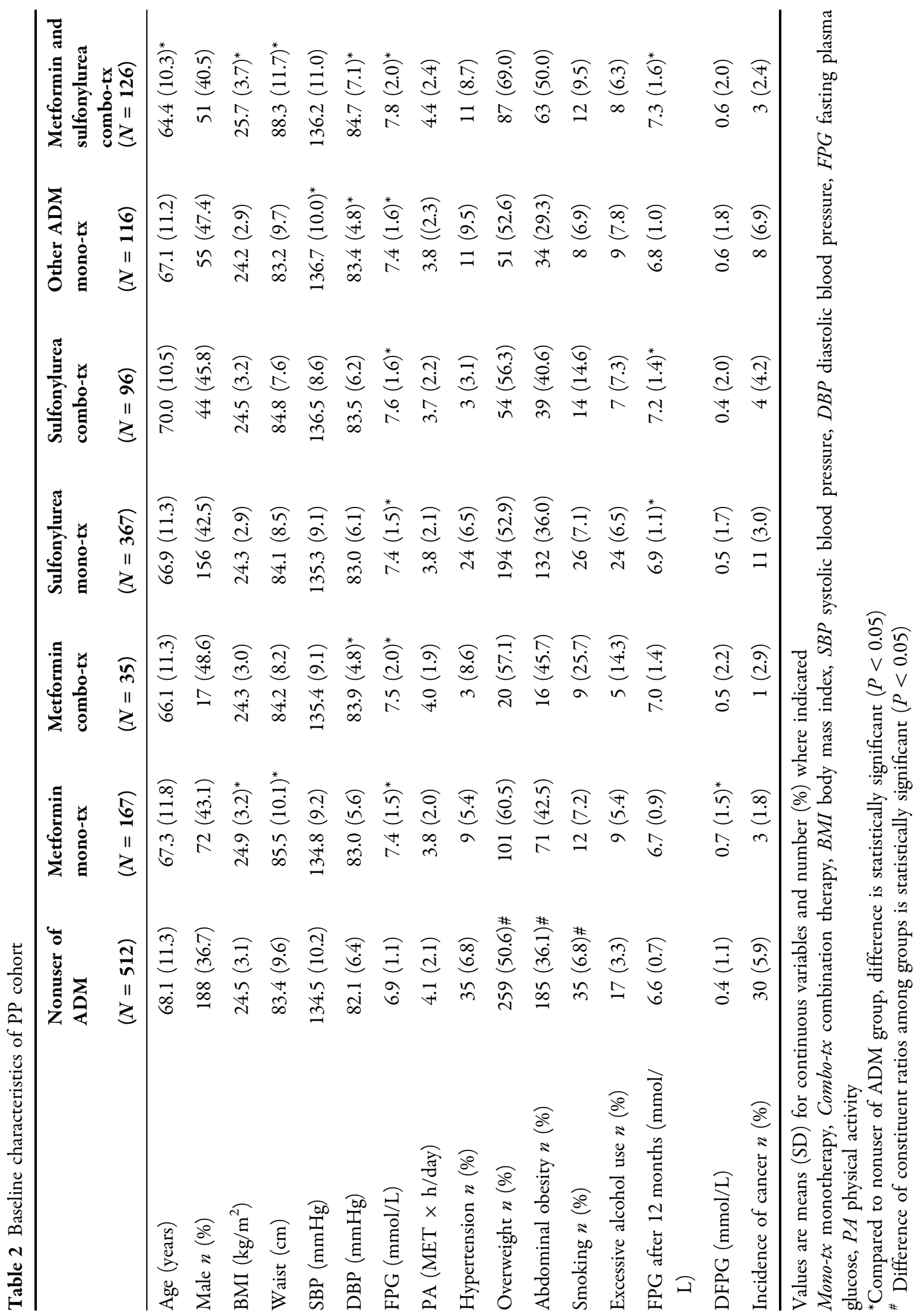


Table 3 Number of cancer cases and rate according to the cancer site

\begin{tabular}{lllrlrr}
\hline Cancer site & ICD10 & ITT cohort & & PP cohort & \\
& & Number of cancer cases & Rate, \% & & Number of cancer cases & Rate, \% \\
\hline Nasopharynx & C11 & 2 & 2.13 & 1 & 1.67 \\
Stomach & C16 & 7 & 7.45 & 4 & 6.67 \\
Small intestine & C17 & 1 & 1.06 & 1 & 1.67 \\
Colorectum & C18-20 & 19 & 20.21 & 9 & 15.00 \\
Liver & C22 & 5 & 5.32 & 4 & 6.67 \\
Gallbladder & C23-24 & 3 & 3.19 & 1 & 1.67 \\
Pancreas & C25 & 4 & 4.26 & 2 & 3.33 \\
Trachea, bronchus, and lung & C34 & 22 & 23.40 & 16 & 26.67 \\
Other skin & C44 & 1 & 1.06 & 1 & 1.67 \\
Breast & C50 & 7 & 7.45 & 7 & 11.67 \\
Corpus uteri & C54 & 1 & 1.06 & 1 & 1.67 \\
Prostate & C61 & 7 & 7.45 & 3 & 5.00 \\
Bladder & C67 & 1 & 1.06 & 0 & 0.00 \\
Brain, central nervous system & C70-72 & 4 & 4.26 & 2 & 3.33 \\
Thyroid & C73 & 7 & 7.45 & 5 & 8.33 \\
Leukemia & C92-95 & 3 & 3.19 & 3 & 5.00 \\
All sites & C00-97 & 94 & 100.00 & 60 & 100.00 \\
\hline
\end{tabular}

\section{Sensitivity Analyses}

Log-rank test was used to assess the differences among nonuser of ADM, monotherapy of metformin, and monotherapy of sulfonylurea groups. Figures 2 and 3 show significant differences of the proportion of cancer-free patients among the groups (Log-rank $=6.374, P=0.041$ in the ITT cohort, Log-rank $=7.14, P=0.028$ in the PP cohort). Table 5 presents the sensitivity results similar to the main analysis.

\section{DISCUSSION}

This study examined a community populationbased retrospective cohort of newly onset T2DM patients. Intention-to treat and per-protocol approaches were applied to analyze the associations of the use of ADM with cancer incidence. We employed ITT and PP approaches to analyze "incident diabetes drug" cohort and ITT analysis can be regarded as the equivalent of a nonrandomized "trial" that avoids many of the biases of traditional observational studies [20]. The results showed that the monotherapy of metformin and monotherapy of sulfonylurea were associated with lower risk of cancer development compared to the nonusers of ADM. Consistent outcomes were also obtained after adjustment for common risk factors.

As the first-line therapy of T2DM, metformin is identified as an insulin-sensitizing agent. In vitro and in vivo studies have provided ample evidence of the antineoplastic effect of metformin. In addition to the direct or indirect effect on lowing the concentration of circulating glucose and insulin, metformin could active 
Table 4 Crude and adjusted HRs for patients with nonuser of ADM versus ADM groups

\begin{tabular}{|c|c|c|c|c|c|c|c|}
\hline \multirow[t]{2}{*}{ Exposure } & \multirow[t]{2}{*}{ Incidence rate $(1000 /$ Pys $)$} & \multicolumn{3}{|c|}{ Crude HR } & \multicolumn{3}{|c|}{ Adjusted $\mathbf{H R}^{\mathbf{a}}$} \\
\hline & & HR & $95 \% \mathrm{CI}$ & $P$ & HR & $95 \% \mathrm{CI}$ & $P$ \\
\hline \multicolumn{8}{|l|}{ ITT cohort } \\
\hline Nonuser of ADM & 11.53 & \multicolumn{3}{|c|}{1.0 (Reference) } & \multicolumn{3}{|c|}{1.0 (Reference) } \\
\hline Metformin mono-tx & 6.61 & 0.58 & $0.30-1.10$ & 0.09 & 0.51 & $0.27-0.99$ & 0.045 \\
\hline Metformin combo-tx & 8.62 & 0.75 & $0.18-3.10$ & 0.69 & 0.60 & $0.15-2.49$ & 0.48 \\
\hline Sulfonylurea mono-tx & 6.27 & 0.55 & $0.32-0.93$ & 0.03 & 0.50 & $0.29-0.85$ & 0.01 \\
\hline Sulfonylurea combo-tx & 7.59 & 0.66 & $0.25-1.85$ & 0.43 & 0.59 & $0.21-1.65$ & 0.31 \\
\hline Other ADM mono-tx & 11.48 & 1 & $0.47-2.13$ & 0.99 & 0.98 & $0.46-2.10$ & 0.96 \\
\hline Metformin and sulfonylurea combo-tx & 5.5 & 0.48 & $0.22-1.02$ & 0.06 & 0.39 & $0.18-0.84$ & 0.02 \\
\hline \multicolumn{8}{|l|}{ PP cohort } \\
\hline Nonuser of $\mathrm{ADM}$ & 12.27 & \multicolumn{3}{|c|}{1.0 (Reference) } & \multicolumn{3}{|c|}{1.0 (Reference) } \\
\hline Metformin mono-tx & 3.73 & 0.31 & $0.09-1.00$ & 0.05 & 0.29 & $0.09-0.96$ & 0.04 \\
\hline Metformin combo-tx & 5.78 & 0.48 & $0.06-3.49$ & 0.47 & 0.41 & $0.06-3.02$ & 0.38 \\
\hline Sulfonylurea mono-tx & 6.15 & 0.51 & $0.25-1.01$ & 0.05 & 0.45 & $0.22-0.91$ & 0.03 \\
\hline Sulfonylurea combo-tx & 8.62 & 0.71 & $0.25-2.00$ & 0.51 & 0.53 & $0.19-1.55$ & 0.25 \\
\hline Other ADM mono-tx & 14.81 & 1.2 & $0.55-2.62$ & 0.65 & 1.14 & $0.52-2.49$ & 0.75 \\
\hline Metformin and sulfonylurea combo-tx & 4.94 & 0.41 & $0.12-1.33$ & 0.14 & 0.32 & $0.09-1.09$ & 0.07 \\
\hline
\end{tabular}

Hazard ratios with 95\% CIs were estimated from Cox proportional hazard models

Mono-tx monotherapy, Combo-tx combination therapy, Pys person-years

a Adjusted for age, gender, BMI, FPG, DFPG, PA, smoking status, and excessive alcohol assumption

the liver enzyme AMPK and inhibit the IGF-1R signaling to suppress the development of cancer $[7,8,21]$. Evidence from epidemiology was inconclusive. Several meta-analyses indicated that metformin use is associated with reduced risk of cancer development compared with other ADMs [9] while some attributed the effect of metformin to biases and confounders [12]. However, there were time-related biases [22] and misclassification of exposure [23] because observational studies were included in the mentioned meta-analyses. These types of biases may lead to underestimation of the risk of cancer in metformin therapy. To avoid the potential time-related bias, some cohort studies preferred the ITT analysis approach [24-26].
Using other ADM therapy group as the comparison, mainly sulfonylurea $[10,25,26]$ and thiazolidinediones [10], these studies and two large randomized trials [10] found no risk association between metformin therapy and cancer. That was consistent with our finding if compared with the sulfonylurea monotherapy group (adjusted HR 1.03, 95\% CI 0.50-2.13). However, in this study, the newly onset T2DM patients were a target population who might prefer a non-pharmaceutical treatment at an early stage of diabetes, although all study subjects were covered by basic medical insurance and community health services in China. In this study, the finding that the metformin monotherapy was associated with lower risk of 


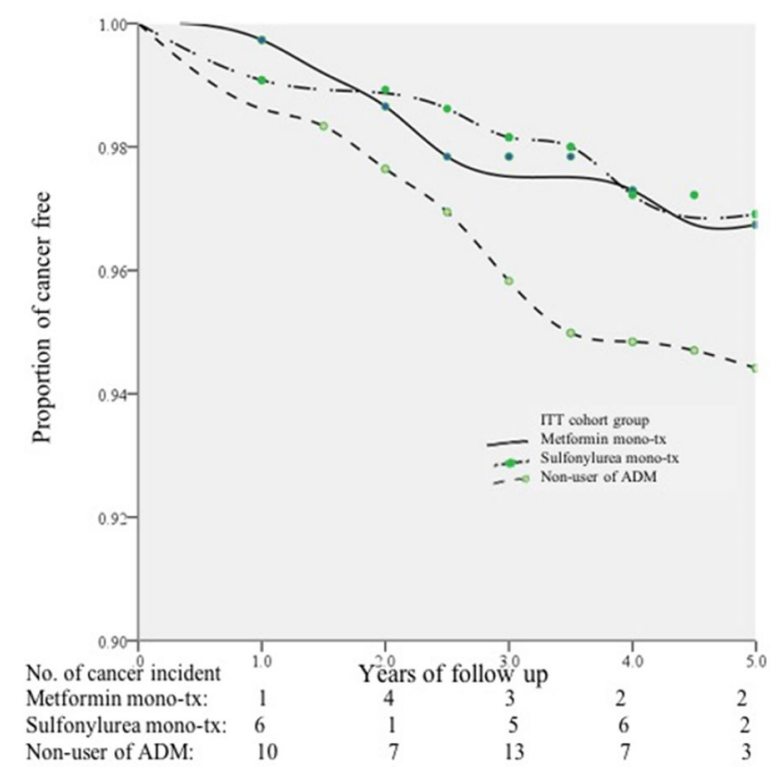

Fig. 2 Proportion of cancer-free patients in the nonuser of ADM group versus metformin and sulfonylurea monotherapy groups in the ITT cohort over 5 years of follow-up

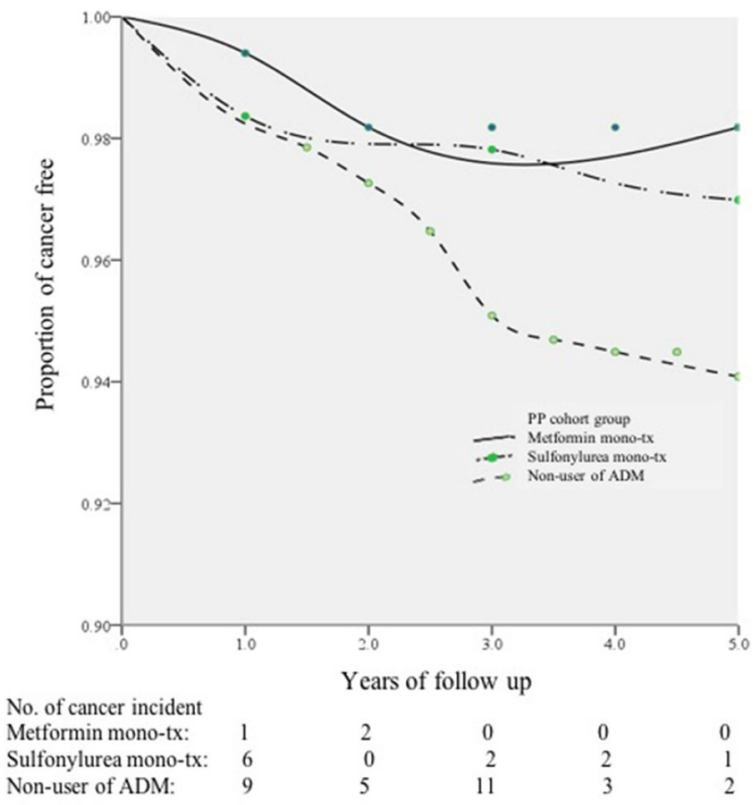

Fig. 3 Proportion of cancer-free patients in the nonuser of ADM group versus metformin and sulfonylurea monotherapy groups in the PP cohort over 5 years of follow-up cancer developing (adjusted HR 0.51,95\% CI 0.27-0.99) compared to nonusers of ADM might suggest that treating newly onset diabetes with metformin will afford greater benefit in reducing risk of developing cancer in the Chinese community T2DM population. However, more RCTs are needed to clarify the causality between metformin and cancer promotion.

On the premise of hyperinsulinemia increasing cancer risk, sulfonylurea as insulin secretagogue seems to be associated with increased risk of cancer. However, our findings suggested that the sulfonylurea might reduce the incidence of cancer (adjusted HR 0.50, 95\% CI 0.29-0.85) compared with nonusers. In observational studies comparing sulfonylureas and metformin, there is exposure time-related bias that may lead to overestimation of the cancer risk effect of sulfonylurea vs. metformin. Our finding is consistent with the findings from other studies which addressed the exposure time-related bias and RCTs. All studies have found that sulfonylurea did not increase the risk of cancer $[10,24,26]$. In this study, the finding that sulfonylurea monotherapy had a similar lower risk of developing cancer compared with metformin monotherapy strategy was also supported by other well-designed ITT studies $[25,26]$.

In order to consider the impact of different ADMs per se on cancer development through glycemic control, the difference between baseline FPG and mean FPG after 12 months was included in the Cox regression model. A positive association of baseline FPG level with cancer incidence [adjusted $\mathrm{HR}=1.35 \quad(95 \%$ CI 1.19-1.54)] and inverse association of DFPG with cancer incidence [adjusted HR $=0.80(95 \%$ CI 0.72-0.90)] level were demonstrated. However, this finding of the potential effect of glucose level on cancer development could not be confirmed by meta-analysis of RCT research [27].

Our study examined a retrospective cohort in an urban district in Shanghai, which may limit the generalizability of the result. The use of information from the Shanghai Community Diabetes Management System of China, which was derived from the periodic GP follow-up and 
Table 5 Risk ratio of cancer incidence of ADM for the different risk factors

\begin{tabular}{|c|c|c|c|c|c|c|}
\hline & \multicolumn{3}{|c|}{ ITT cohort } & \multicolumn{3}{|c|}{ PP cohort } \\
\hline & \multicolumn{3}{|c|}{ Adjusted HR $^{a}$} & \multicolumn{3}{|c|}{ Adjusted $H^{a}$} \\
\hline & HR & $95 \% \mathrm{CI}$ & $P$ & HR & $95 \% \mathrm{CI}$ & $P$ \\
\hline Age (years) & 1.02 & $1.00-1.04$ & 0.03 & 1.02 & $1.0-1.05$ & 0.06 \\
\hline \multicolumn{7}{|l|}{ Gender } \\
\hline Male & \multicolumn{3}{|c|}{1.0 (Reference) } & \multicolumn{3}{|c|}{1.0 (Reference) } \\
\hline Female & 0.97 & $0.62-1.50$ & 0.87 & 1.27 & $0.73-2.22$ & 0.4 \\
\hline BMI $\left(\mathrm{kg} / \mathrm{m}^{2}\right)$ & 1.01 & $0.95-1.07$ & 0.42 & 1.03 & $0.95-1.12$ & 0.48 \\
\hline Baseline FPG $(\mathrm{mmol} / \mathrm{L})$ & 1.35 & $1.19-1.54$ & $<0.001$ & 1.3 & $1.07-1.58$ & 0.01 \\
\hline DFPG $(\mathrm{mmol} / \mathrm{L})$ & 0.8 & $0.72-0.90$ & $<0.001$ & 0.77 & $0.67-0.87$ & $<0.001$ \\
\hline PA $($ MET $\times$ h/day $)$ & 0.99 & $0.90-1.09$ & 0.88 & 1.01 & $0.89-1.14$ & 0.92 \\
\hline \multicolumn{7}{|l|}{ Smoking status } \\
\hline Never & \multicolumn{3}{|c|}{1.0 (Reference) } & \multicolumn{3}{|c|}{1.0 (Reference) } \\
\hline Ever & 1.3 & $0.69-2.46$ & 0.42 & 1.42 & $0.61-3.31$ & 0.42 \\
\hline \multicolumn{7}{|l|}{ Excess alcohol assumption } \\
\hline Never & \multicolumn{3}{|c|}{1.0 (Reference) } & \multicolumn{3}{|c|}{1.0 (Reference) } \\
\hline Ever & 1.01 & $0.40-2.54$ & 0.99 & 0.95 & $0.27-3.36$ & 0.93 \\
\hline \multicolumn{7}{|l|}{$\mathrm{ADM}$} \\
\hline Nonuser of ADM & \multicolumn{3}{|c|}{1.0 (Reference) } & \multicolumn{3}{|c|}{1.0 (Reference) } \\
\hline Metformin mono-tx & 0.51 & $0.27-0.99$ & 0.045 & 0.29 & $0.09-0.96$ & 0.04 \\
\hline Metformin combo-tx & 0.6 & $0.15-2.49$ & 0.48 & 0.41 & $0.06-3.02$ & 0.38 \\
\hline Sulfonylurea mono-tx & 0.5 & $0.29-0.85$ & 0.01 & 0.45 & $0.22-0.91$ & 0.03 \\
\hline Sulfonylurea combo-tx & 0.59 & $0.21-1.65$ & 0.31 & 0.53 & $0.19-1.55$ & 0.25 \\
\hline Other ADM mono-tx & 0.98 & $0.46-2.10$ & 0.96 & 1.14 & $0.52-2.49$ & 0.75 \\
\hline Metformin and sulfonylurea combo-tx & 0.39 & $0.18-0.84$ & 0.02 & 0.32 & $0.09-1.09$ & 0.07 \\
\hline
\end{tabular}

outpatient visits, might minimize recall bias and improve the adherence. This study does not control socioeconomic status of the patients, which might confound the relationship between medication use and cancer rates. However, almost all of the patients were covered by urban basic medical insurance and managed by community health service centers, which ensure their access to essential medications. Also, individuals included in this study came from the same district of Shanghai
Municipality, which may have reduced the heterogeneity in socioeconomic status among residents. Third, the small sample size also did not allow us to analyze the associations between $\mathrm{ADM}$ and the risk of particular cancer sites. As a result of accessibility and integrity in blood glucose level of the current cohort data source, we only extracted the FPG information. Other indicators for glucose level such as HbA1c and postprandial plasma glucose should be included in future research with better accessibility and 
Table 6 HRs for patients in nonuser of ADM versus ADM groups in subgroup sensitivity analyses

\begin{tabular}{|c|c|c|c|}
\hline & HR & 95\% CI & $P$ \\
\hline \multicolumn{4}{|l|}{ Gender } \\
\hline Male & \multicolumn{3}{|c|}{1.0 (Reference) } \\
\hline Female & 0.94 & $0.57-1.55$ & 0.8 \\
\hline \multicolumn{4}{|l|}{ Age (years) } \\
\hline$<65$ & \multicolumn{3}{|c|}{1.0 (Reference) } \\
\hline$\geq 65$ & 1.73 & $1.05-2.84$ & 0.03 \\
\hline \multicolumn{4}{|l|}{$\mathrm{FPG}(\mathrm{mmol} / \mathrm{L})$} \\
\hline$<7.2$ & \multicolumn{3}{|c|}{1.0 (Reference) } \\
\hline$\geq 7.2$ & 1.45 & $0.90-2.32$ & 0.13 \\
\hline \multicolumn{4}{|l|}{$\operatorname{BMI}\left(\mathrm{kg} / \mathrm{m}^{2}\right)$} \\
\hline$<24$ & \multicolumn{3}{|c|}{1.0 (Reference) } \\
\hline$\geq 24$ & 0.93 & $0.55-1.56$ & 0.78 \\
\hline \multicolumn{4}{|l|}{ Waist circumstance $(\mathrm{cm})$} \\
\hline Male $<90$ or female $<80$ & \multicolumn{3}{|c|}{1.0 (Reference) } \\
\hline Male $\geq 90$ or female $\geq 80$ & 1.14 & $0.67-1.92$ & 0.63 \\
\hline \multicolumn{4}{|l|}{ Smoking status } \\
\hline Never & \multicolumn{3}{|c|}{1.0 (Reference) } \\
\hline Ever & 1.64 & $0.74-3.61$ & 0.22 \\
\hline \multicolumn{4}{|l|}{ Excess alcohol assumption } \\
\hline Never & \multicolumn{3}{|c|}{1.0 (Reference) } \\
\hline Ever & 0.61 & $0.18-2.10$ & 0.43 \\
\hline \multicolumn{4}{|l|}{ Adherence } \\
\hline Non-switch & \multicolumn{3}{|c|}{1.0 (Reference) } \\
\hline Switch & 1.07 & $0.66-1.74$ & 0.78 \\
\hline \multicolumn{4}{|l|}{$\mathrm{ADM}$} \\
\hline Nonuser & \multicolumn{3}{|c|}{1.0 (Reference) } \\
\hline Metformin mono-tx & 0.55 & $0.29-1.07$ & 0.08 \\
\hline Sulfonylurea mono-tx & 0.52 & $0.30-0.90$ & 0.02 \\
\hline
\end{tabular}

Hazard ratios with $95 \%$ CIs were estimated from Cox proportional hazard models

Mono-tx monotherapy, Combo-tx combination therapy data quality. The effects on cancer sites might not be equivalent for metformin $[28,29]$. There might not be equivalent effects among generations of sulfonylureas. Considering the dose effect, there was a lack of information on cumulative dose of medication. Lastly, there might be still unobservable confounding factors; however, in our study, common risk factors for cancer, like overweight, physical activity, smoking, and alcohol assumption, were considered and adjusted for in the model.

\section{CONCLUSION}

In our study, the use of metformin or sulfonylurea might be associated with lower incidence of cancer in a cohort of newly onset T2DM patients in China. More studies with larger sample size are needed to examine the associations between antidiabetic medications and incidence of cancer by sites.

\section{ACKNOWLEDGEMENTS}

The authors would like to thank all the investigators and patients in the study.

Funding. Sponsorship for this study and the article processing charges were funded by The Fourth Round of Shanghai Three-year Action Plan on Public Health Discipline and Talent Program: Evidence-based Public Health and Health Economics (No. 15GWZK0901), The Youth Scientific Project of Shanghai Municipal Commission of Health and Family Planning (No. 20154Y0193) and Key Project of Shanghai Hongkou Commission of Health and Family Planning (Hongwei 1801-02).

Authorship. All named authors meet the International Committee of Medical Journal Editors (ICMJE) criteria for authorship for this manuscript, take responsibility for the integrity of the work as a whole, and have given final approval to the version to be published. 
Prior Presentation. The data were partially presented at the ISPOR 22nd Annual International Meeting in Boston 20-24 May, 2017; reference: Value in Health, 2017; 20 (5): A167 (abstract).

Disclosures. Jing-Hong Ye, Meng-Hua Qian, Li-Zheng Shi and $\mathrm{Lu}$ Ye have nothing to disclose.

Compliance with Ethics Guidelines. The study design was approved by the Medical Ethics Committee of Fudan University, School of Public Health (reference numbers IRB00002408 and FWA00002399). All procedures performed in studies involving human patients were in accordance with the ethical standards of the institutional and/or national research committee and with the 1964 Helsinki declaration and its later amendments or comparable ethical standards. Informed consent was obtained from all individual patients included in the study.

Data Availability. The data sets generated and/or analyzed during the current study are available from the corresponding author on reasonable request.

Open Access. This article is distributed under the terms of the Creative Commons Attribution-NonCommercial 4.0 International License (http://creativecommons.org/licenses/ by-nc/4.0/), which permits any noncommercial use, distribution, and reproduction in any medium, provided you give appropriate credit to the original author(s) and the source, provide a link to the Creative Commons license, and indicate if changes were made.

\section{REFERENCES}

1. Cho NH, Whiting D, Forouhi N, et al. IDF diabetes Atlas. 7th ed. Brussels: Karakas; 2015. p. 144.

2. World Health Organization. Cancer fact sheet 2015. https://www.who.int/mediacentre/factsheets/fs297/ en/. Accessed Feb 2017

3. Noto H, Tsujimoto T, Noda M. Significantly increased risk of cancer in diabetes mellitus patients: a meta-analysis of epidemiological evidence in Asians and non-Asians. J Diabetes Investig. 2012;3(1):24-33.

4. Campbell PT, Newton CC, Patel AV, Jacobs EJ, Gapstur SM. Diabetes and cause-specific mortality in a prospective cohort of one million U.S. adults. Diabetes Care. 2012;35(9):1835-44.

5. Hotamisligil GS. Inflammation and metabolic disorders. Nature. 2006;444(7121):860-7.

6. Nasser MW, Wani NA, Ahirwar DK, et al. RAGE mediates S100A7-induced breast cancer growth and metastasis by modulating the tumor microenvironment. Cancer Res. 2015;75(6):974-85.

7. Cerezo M, Tichet M, Abbe P, et al. Metformin blocks melanoma invasion and metastasis development in AMPK/p53-dependent manner. Mol Cancer Ther. 2013;12(8):1605-15.

8. Cao H, Dong W, Qu X, et al. Metformin enhances the therapy effects of anti-IGF-1R mAb figitumumab to NSCLC. Sci Rep. 2016;6:31072.

9. Thakkar B, Aronis KN, Vamvini MT, Shields K, Mantzoros CS. Metformin and sulfonylureas in relation to cancer risk in type II diabetes patients: a meta-analysis using primary data of published studies. Metabolism. 2013;62(7):922-34.

10. Home PD, Kahn SE, Jones NP, et al. Experience of malignancies with oral glucose-lowering drugs in the randomised controlled ADOPT (A Diabetes Outcome Progression Trial) and RECORD (Rosiglitazone Evaluated for Cardiovascular Outcomes and Regulation of Glycaemia in Diabetes) clinical trials. Diabetologia. 2010;53(9):1838-45.

11. Bonanni B, Puntoni M, Cazzaniga M, et al. Dual effect of metformin on breast cancer proliferation in a randomized presurgical trial. J Clin Oncol. 2012;30(21):2593-600.

12. Gandini S, Puntoni M, Heckman-Stoddard BM, et al. Metformin and cancer risk and mortality: a systematic review and meta-analysis taking into account biases and confounders. Cancer Prev Res (Phila). 2014;7(9):867-85.

13. Zhang L, Ji L, Guo L, et al. Treatment patterns and glycemic control in older adults with type 2 diabetes mellitus receiving only oral antidiabetes drugs in China. Diabetes Technol Ther. 2015;17(11):816-24.

14. Tuccori M, Wu JW, Yin H, Majdan A, Azoulay L. The use of glyburide compared with other sulfonylureas and the risk of cancer in patients with type 2 diabetes. Diabetes Care. 2015;38(11):2083-9. 
15. Gu Q, Wang C, Wang G, et al. Glipizide suppresses embryonic vasculogenesis and angiogenesis through targeting natriuretic peptide receptor A. Exp Cell Res. 2015;333(2):261-72.

16. Qi C, Zhou Q, Li B, et al. Glipizide, an antidiabetic drug, suppresses tumor growth and metastasis by inhibiting angiogenesis. Oncotarget. 2014;5(20): 9966-79.

17. Chinese Diabetes Society. China guideline for type 2 diabetes (2013). Chin J Diab. 2014;6(7):447-98.

18. Sallis JF, Haskell WL, Wood PD, et al. Physical activity assessment methodology in the five-city project. Am J Epidemiol. 1985;121(1):91-106.

19. American Diabetes Association. Standards of medical care in diabetes-2015. Diabetes Care. 2015;38(Suppl):S1-S2.

20. Hernan MA, Alonso A, Logan R, et al. Observational studies analyzed like randomized experiments: an application to postmenopausal hormone therapy and coronary heart disease. Epidemiology. 2008;19(6):766-79.

21. Li L, Wang YB, Peng T, et al. Metformin restores crizotinib sensitivity in crizotinib-resistant human lung cancer cells through inhibition of IGF1-R signaling pathway. Oncotarget. 2016;7(23):34442-52.

22. Suissa S, Azoulay L. Metformin and the risk of cancer: time-related biases in observational studies. Diabetes Care. 2012;35(12):2665-733.
23. Stricker BH, Stijnen T. Analysis of individual drug use as a time-varying determinant of exposure in prospective population-based cohort studies. Eur J Epidemiol. 2010;25(4):245-51.

24. But A, Wang H, Mannisto S, Pukkala E, Haukka J. Assessing the effect of treatment duration on the association between anti-diabetic medication and cancer risk. PLoS One. 2014;9(11):e113162.

25. Tsilidis KK, Capothanassi D, Allen NE, et al. Metformin does not affect cancer risk: a cohort study in the U.K. Clinical Practice Research Datalink analyzed like an intention-to-treat trial. Diabetes Care. 2014;37(9):2522-32.

26. Kowall B, Rathmann W, Kostev K. Are sulfonylurea and insulin therapies associated with a larger risk of cancer than metformin therapy? A retrospective database analysis. Diabetes Care. 2015;38(1): 59-655.

27. Johnson JA, Bowker SL. Intensive glycaemic control and cancer risk in type 2 diabetes: a meta-analysis of major trials. 2011;54(1):25-31.

28. Luo J, Phillips L, Liu S, Wactawski-Wende J, Margolis KL. Diabetes, diabetes treatment, and risk of thyroid cancer. J Clin Endocrinol Metab. 2016;101(3):1243-8.

29. He XK, Su TT, Si JM, Sun LM. Metformin is associated with slightly reduced risk of colorectal cancer and moderate survival benefits in diabetes mellitus: a metaanalysis. Medicine (Baltimore). 2016;95(7):e2749. 